\title{
Next-generation sequencing in neuropathologic diagnosis of infections of the nervous system
}

\section{OPEN}

Steven L. Salzberg, PhD

Florian P. Breitwieser,

$\mathrm{PhD}$

Anupama Kumar, MBBS

Haiping Hao, $\mathrm{PhD}$

Peter Burger, MD

Fausto J. Rodriguez, MD

Michael Lim, MD

Alfredo Quiñones-

Hinojosa, MD

Gary L. Gallia, MD

Jeffrey A. Tornheim, MD

Michael T. Melia, MD

Cynthia L. Sears, MD

Carlos A. Pardo, MD

Correspondence to

Dr. Pardo:

cpardov1@jhmi.edu
Supplemental data at Neurology.org/nn

\section{ABSTRACT}

Objective: To determine the feasibility of next-generation sequencing (NGS) microbiome approaches in the diagnosis of infectious disorders in brain or spinal cord biopsies in patients with suspected CNS infections.

Methods: In a prospective pilot study, we applied NGS in combination with a new computational analysis pipeline to detect the presence of pathogenic microbes in brain or spinal cord biopsies from 10 patients with neurologic problems indicating possible infection but for whom conventional clinical and microbiology studies yielded negative or inconclusive results.

Results: Direct DNA and RNA sequencing of brain tissue biopsies generated 8.3 million to 29.1 million sequence reads per sample, which successfully identified with high confidence the infectious agent in 3 patients for whom validation techniques confirmed the pathogens identified by NGS. Although NGS was unable to identify with precision infectious agents in the remaining cases, it contributed to the understanding of neuropathologic processes in 5 others, demonstrating the power of large-scale unbiased sequencing as a novel diagnostic tool. Clinical outcomes were consistent with the findings yielded by NGS on the presence or absence of an infectious pathogenic process in 8 of 10 cases, and were noncontributory in the remaining 2 .

Conclusions: NGS-guided metagenomic studies of brain, spinal cord, or meningeal biopsies offer the possibility for dramatic improvements in our ability to detect (or rule out) a wide range of CNS pathogens, with potential benefits in speed, sensitivity, and cost. NGS-based microbiome approaches present a major new opportunity to investigate the potential role of infectious pathogens in the pathogenesis of neuroinflammatory disorders. Neurol Neuroimmunol Neuroinflamm 2016;3:e251; doi: 10.1212/NXI.0000000000000251

\section{GLOSSARY}

EBV = Epstein-Barr virus; FIRES $=$ febrile infection-related epilepsy syndrome; JCV = JC polyomavirus; NGS = nextgeneration sequencing; $\mathbf{P M L}=$ progressive multifocal leukoencephalopathy.

Ascertainment of the etiology of inflammatory disorders of the CNS represents a major challenge in the clinical setting, as more than $50 \%$ of cases go undiagnosed. ${ }^{1}$ Next-generation sequencing (NGS) and metagenomics present a major new opportunity to investigate the potential role of infection in the pathogenesis of neuroinflammatory disorders. This technology can provide a view of the transcriptome of the host tissue as well as capture microbial genomes (i.e., bacteria, fungi, and viruses) that reside in the tissue niche. ${ }^{2-4}$ Until recently, most sequencebased pathogen identification studies have focused on targeted capture of the 16S rRNA gene that is exclusive to prokaryotes. Deep sequencing of total DNA or RNA provides an unbiased approach that can detect even rare components of the microbiome. ${ }^{5}$ This strategy has recently been used to diagnose cases of encephalitis and meningitis by known and novel pathogens, ${ }^{6-15}$

\footnotetext{
From the Center for Computational Biology, McKusick-Nathans Institute of Genetic Medicine (S.L.S., F.P.B.), Department of Neurology (A.K., C.A.P.), Deep Sequencing and Microarray Core (H.H.), and Departments of Pathology (P.B., F.J.R., C.A.P.), Neurosurgery (M.L., A.Q.-H., G.L.G.), and Medicine (J.A.T., M.T.M., C.L.S.), School of Medicine, and Departments of Biomedical Engineering, Computer Science, and Biostatistics (S.L.S.), Johns Hopkins University, Baltimore, MD.

Funding information and disclosures are provided at the end of the article. Go to Neurology.org/nn for full disclosure forms. The Article Processing Charge was paid by the authors.

This is an open access article distributed under the terms of the Creative Commons Attribution-NonCommercial-NoDerivatives License 4.0 (CC BY-NC-ND), which permits downloading and sharing the work provided it is properly cited. The work cannot be changed in any way or used commercially.
} 


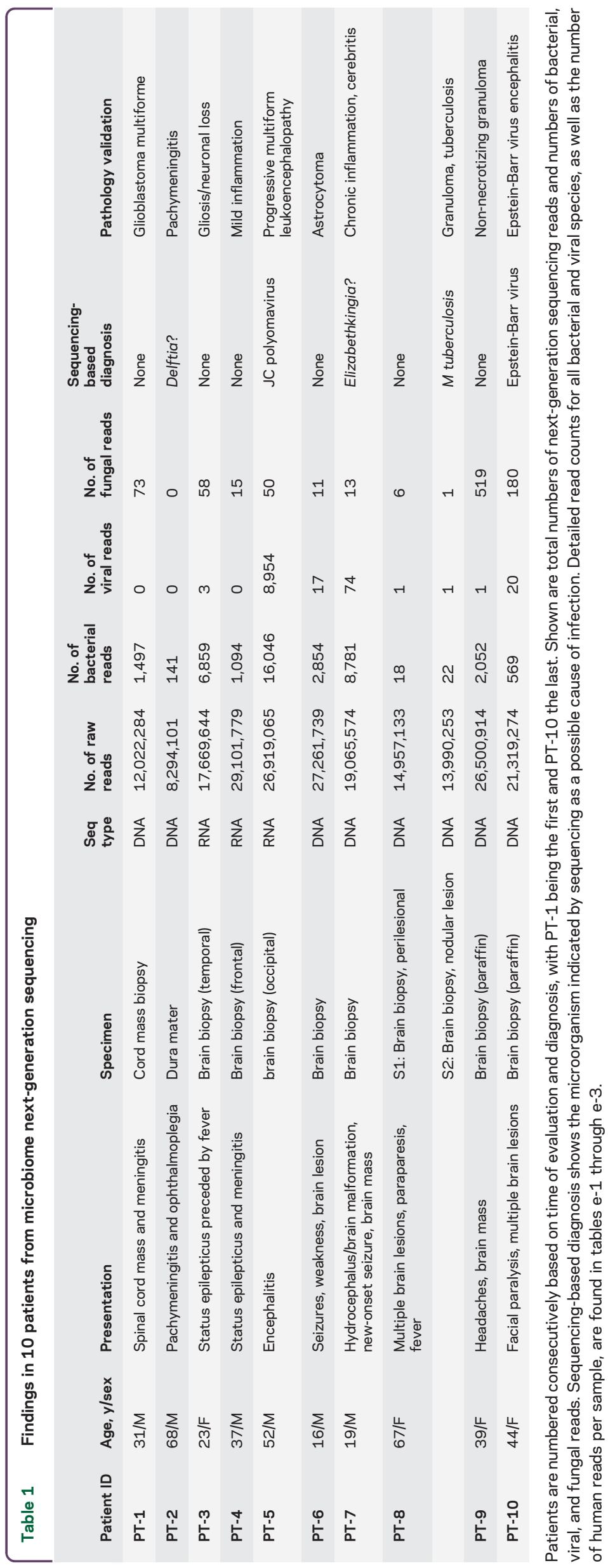

but other than these isolated cases, the utility of NGS for clinical or pathologic diagnosis has yet to be established.

We report a pilot prospective study of the use of unbiased NGS to assist in the evaluation of brain biopsies in a series of patients with neuroinflammatory disorders suspected to be associated with infections. We applied NGS and a new computational analysis pipeline for identifying pathogen species based on short NGS reads, as short as 100 base pairs (bp) in length. ${ }^{16}$

METHODS Patients, biopsy handling, and sequencing. CNS tissues were obtained prospectively from biopsies performed during diagnostic assessment of 10 patients with neuroinflammatory disorders (table 1). Fresh frozen tissues from 8 cases were sequenced immediately after biopsy and 2 other samples were from paraffin-processed tissues (e-Methods at Neurology.org/nn).

Standard protocol approvals, registrations, and patient consents. All biopsy tissues were obtained from a biosample repository approved by The Johns Hopkins University School of Medicine Institutional Review Board.

Computational processing. All reads were run through the Kraken system, ${ }^{16}$ which compared them to a database containing the human genome (version GRCh38.p2), 2,817 bacterial genomes (representing 891 distinct species), 4,383 viral genomes (2,963 species), and 26 genomes of eukaryotic pathogens. The total size of the Kraken database in this study was 97 gigabytes. Each Kraken report was analyzed separately, and reads matching potential causative agents (bacteria or viruses) were extracted from the sequence file and realigned using the more sensitive BLAST $^{17}$ aligner against the comprehensive NCBI nucleotide database (nt), which contains many thousands of draft genomes and partial sequences in addition to the finished genomes in the custom Kraken database used here. In all cases, computational analysts were blinded to all pathology results until the analysis was complete, and in most cases NGS analysis was conducted in parallel with pathologic studies. Only reads whose best BLAST matches hit the same species were considered further. Reads that hit different species were considered uninformative and were excluded from further analysis. For all of the positive diagnoses reported here, the BLAST results confirmed the species identified by Kraken, although the best-matching strain was sometimes a different (draft) genome (see e-Methods for additional details). All nonhuman reads have been deposited in the NCBI Sequence Read Archive under project PRJNA314149.

RESULTS Sequencing data. The sequenced biopsies (appendix e-1) generated between 8.3 and 29.1 million reads per sample (table 1 ). The vast majority of reads in all cases were human, as expected (because the human genome is approximately 1,000 times larger than most bacterial genomes, a mixture with equal numbers of human and bacterial cells will yield 99.9\% human DNA). After filtering to remove common contaminants and vector sequences, we then ranked the microbial species and considered only those that ranked among the top 3 species as possible infectious agents 
(figure 1). The number of bacterial and viral reads varied from fewer than 20 to 25,000 . NGS successfully identified with high confidence the infectious agent in 3 out of 10 patients. In 2 cases the NGS cases were indeterminate and the other remaining 5 patients had negative or nonspecific findings, which further supported the conclusion that the disease process was not associated with active infection. In 2 cases, diagnoses of noninfectious processes (e.g., gliomas) were identified by pathology.

Cases with a high degree of diagnostic confidence and positive pathogen identification. Patient PT-8 (pathogen: Mycobacterium tuberculosis). A 67-year-old woman with osteomyelitis and lung disease presented with multifocal brain and spinal lesions (appendix e-2). Brain MRI showed progression of multiple nodular enhancing lesions throughout the brain (figure 2). Biopsies from a perilesional (S1) and a nodular brain lesion (S2) were obtained for pathology, microbiology, and NGS studies. Two DNA sequencing runs yielded $15 \mathrm{M}$ reads from sample S1 and $14 \mathrm{M}$ reads from sample S2. These runs yielded the fewest microbial reads of any of the patients in our study: 18 and 22 bacterial reads, and only 1 to 6 viral and fungal reads, respectively, for samples S1 and S2. Nonetheless, a clear finding emerged for sample S2: 15 reads from $M$ tuberculosis. Despite the small absolute number of reads, this species explained $68 \%$ of the bacterial reads detected. We manually confirmed the sequence assignments using BLAST $^{17}$ to align them against the NCBI nt database. We then realigned all reads against one specific genome, $M$ tuberculosis 7199-99 (accession NC_020089.1), using Bowtie2 with sensitive local alignment settings. ${ }^{18}$ This

Figure 1 Heatmap shows the top microbial species in each of the 11 samples

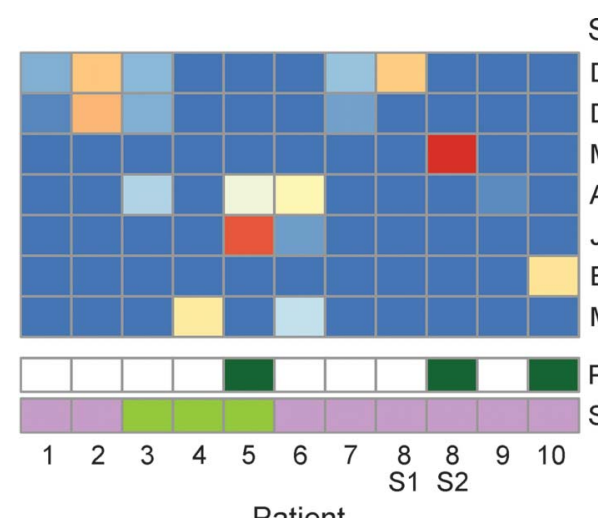

Species

Delftia acidovorans

Delftia sp. Cs1-4

Mycobacterium tuberculosis complex

Akkermansia muciniphila

JC polyomavirus

Epstein-Barr virus

Mycoplasma hyorhinis

Positive pathogen identification

Sequencing type: DNA, RNA

Patient

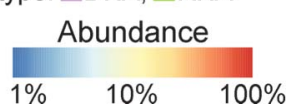

All microbial species that explain $10 \%$ or more of microbial reads (excluding contaminants) in any sample are shown. Shaded squares indicate the relative proportion of reads from each species in that sample, ranging from blue (1\%) to red (100\%). Green boxes across the bottom indicate the 3 samples for which the sequence-based diagnosis was independently confirmed. procedure yielded 34 reads that were randomly distributed along the $M$ tuberculosis genome. Pathology studies of the corresponding S2 sample showed necrotizing granulomas although microbiologic studies failed to identify any microorganism (figure 2). The patient responded rapidly to antituberculous treatment.

Patient PT-5 (pathogen: JC polyomavirus [JCV]). A 52year-old man had been admitted for evaluation of lower extremity weakness, right hemiparesis, and a simple partial motor seizure. A brain MRI showed a left postcentral frontal cortical and white matter lesion (figure 3A). PCR CSF studies for viruses and bacterial cultures were negative although a prebiopsy CSF PCR was reported inconclusive or low DNA levels for JCV. A biopsy of the lesion underwent RNA sequencing, which yielded 26.9 million reads, of which 25.9 million were human (appendix e-1). Analysis found JCV with 8,944 out of 8,954 reads from all viruses. Although many bacterial species were detected, JCV was the most abundant species in terms of the number of reads, despite its small genome size. The whole genome of JCV was covered by the reads, at an average depth over 200 (figure 3B). The sequence data supported the diagnosis of progressive multifocal leukoencephalopathy (PML). ${ }^{19}$ Pathology results showed marked astrogliosis and intranuclear inclusions in oligodendrocytes (figure 3C) and positive immunostaining for SV40 T antigen (a surrogate for JCV), confirming the diagnosis (figure 3D).

Patient PT-10 (pathogen: Epstein-Barr virus [EBV]). A 44-year-old woman with a history of organ transplants and immunosuppression presented with facial paralysis. Brain MRI showed at least 3 enhancing lesions in both hemispheres, one of them with appearance of a ring-enhancing lesion, which resembled CNS toxoplasmosis (figure 4, A-C). Assessment of CSF for known opportunistic infections was negative as well as CSF flow cytometry for malignancies. Biopsy of one lesion showed granulomatous, lymphohistocytic inflammation and focal necrosis (figure 4D). Paraffin sections were processed for NGS, which yielded 21.3 million reads (table 1 ), of which 21 million were human and $\sim 216,000$ were vector or synthetic controls. Only 569 reads were bacteria, all matching known skin bacteria or contaminants. Twenty reads matched viruses, of which 18 (90\%) matched EBV. A validation test using in situ hybridization for EBV-encoded RNA confirmed EBV infection (figure $4 \mathrm{E}$ ).

Cases with indeterminate pathogen identification (not confirmed by a second method). Patient PT-2 with a Tolosa-Hunt-like syndrome and focal pachymeningitis. A 69-year-old man developed left-sided ptosis and 

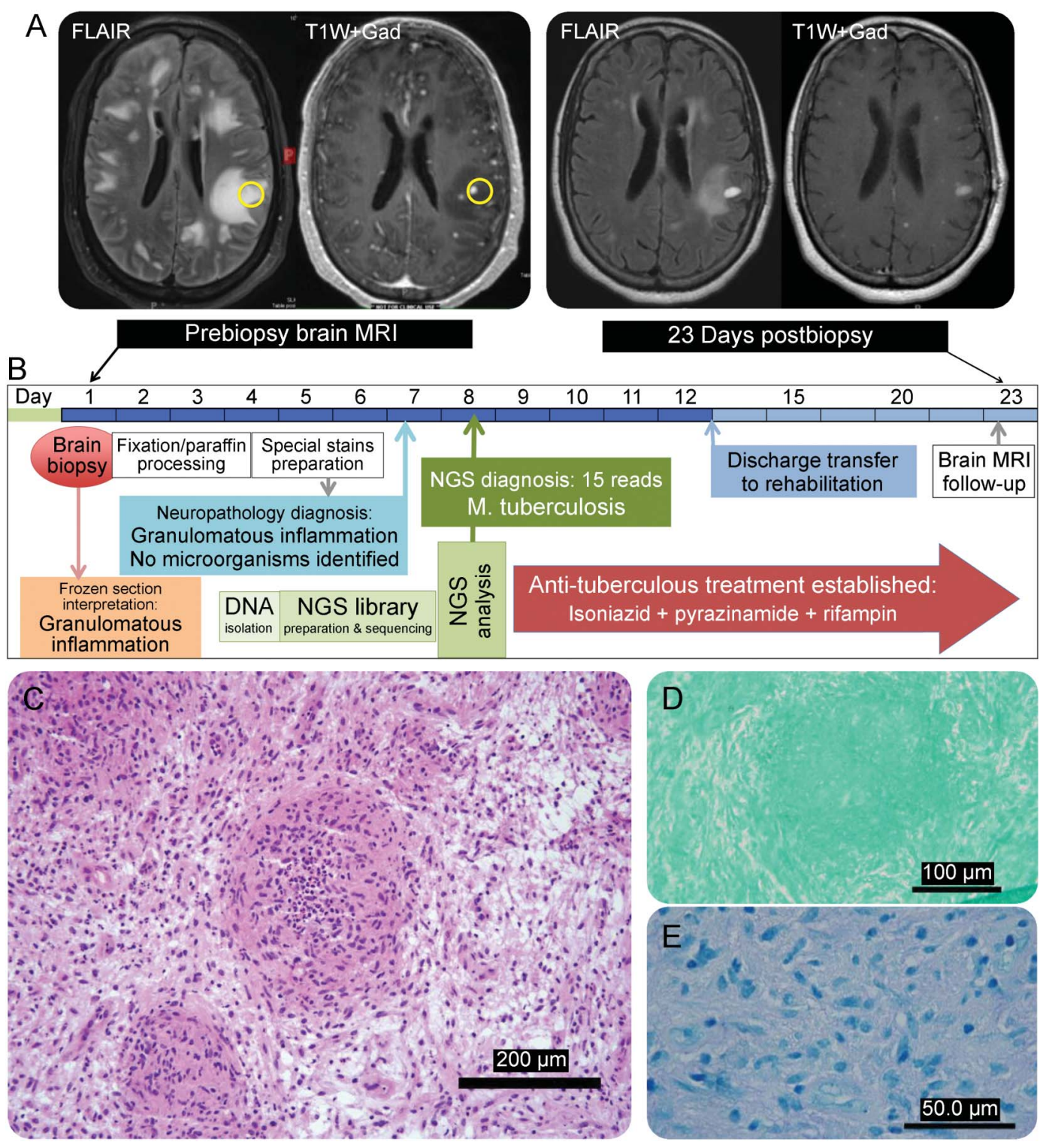

(A) Prebiopsy brain MRI in patient PT-8 shows multifocal subcortical and deep white matter lesions in fluid-attenuated inversion recovery (FLAIR) MRI. Several focal nodular enhancing lesions are seen in T1-weighted + gadolinium (Gad) MRI. A brain biopsy targeted a nodular lesion in the left posterior frontal-parietal region (yellow circles). Subsequent brain MRIs demonstrate a dramatic improvement of the lesions 23 days after biopsy and 15 days after instauration of the anti-TB medications. (B) Diagrammatic representation of the time profile for biopsy processing for neuropathology and next-generation sequencing (NGS) diagnosis in PD-8. Although histologic studies were able to establish the presence of a granulomatous inflammation immediately after biopsy, a more comprehensive neurohistologic analysis completed 6 days after biopsy failed to demonstrate infectious pathogens. NGS diagnosis was established 7 days after biopsy, confirming the presence of Mycobacterium tuberculosis, a finding that was used to support the treatment with antituberculous medication and ruled out the presence of Nocardia infection. (C) Histologic demonstration of granulomatous inflammation in hematoxylin \& eosin stains. (D) Grocott methenamine silver stain is negative for fungal species. Similarly, Ziehl-Neelsen stain for acid-fast bacilli (E) is negative.

retro-orbital headache after cataract and lens implantation surgeries. The symptoms were followed by decreased vision, diplopia, ophthalmoplegia, and facial numbness. He was diagnosed with Tolosa-Hunt syndrome and treated with dexamethasone. Brain MRIs demonstrated pachymeningeal enhancement localized in the medial aspect of the left middle cranial fossae, left cavernous sinus, and adjacent structures (figure 5A), which worsened over time despite steroid treatment. A biopsy of the dural and skull base mass showed chronic inflammation and fibrosis (figure 5C). Microbiologic studies were negative. NGS studies of the dura biopsy were indeterminate although a high proportion of Delftia acidovorans (or possibly Chryseobacterium taeanense, which is very closely related to Delftia) and 

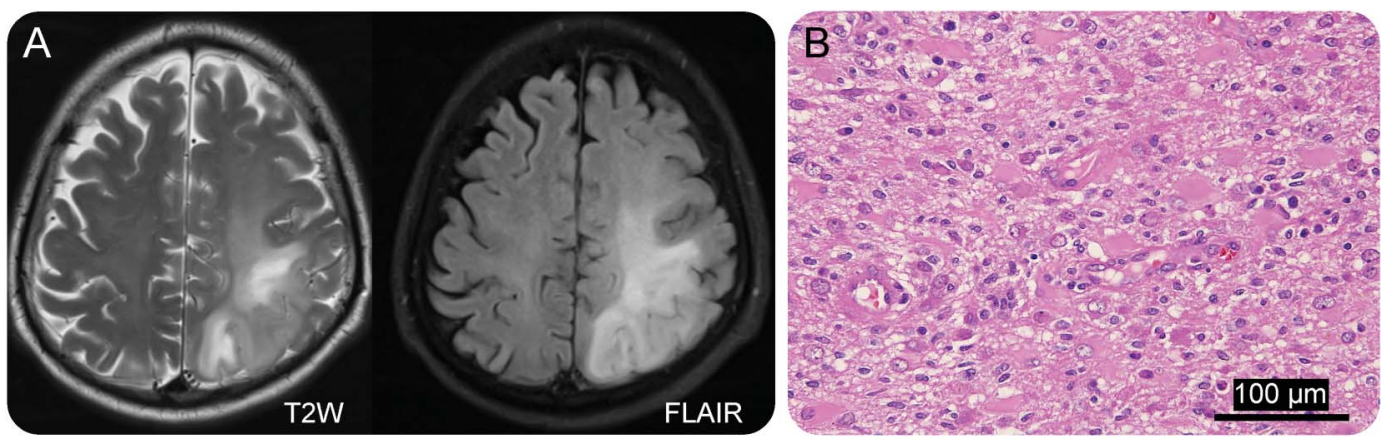

C
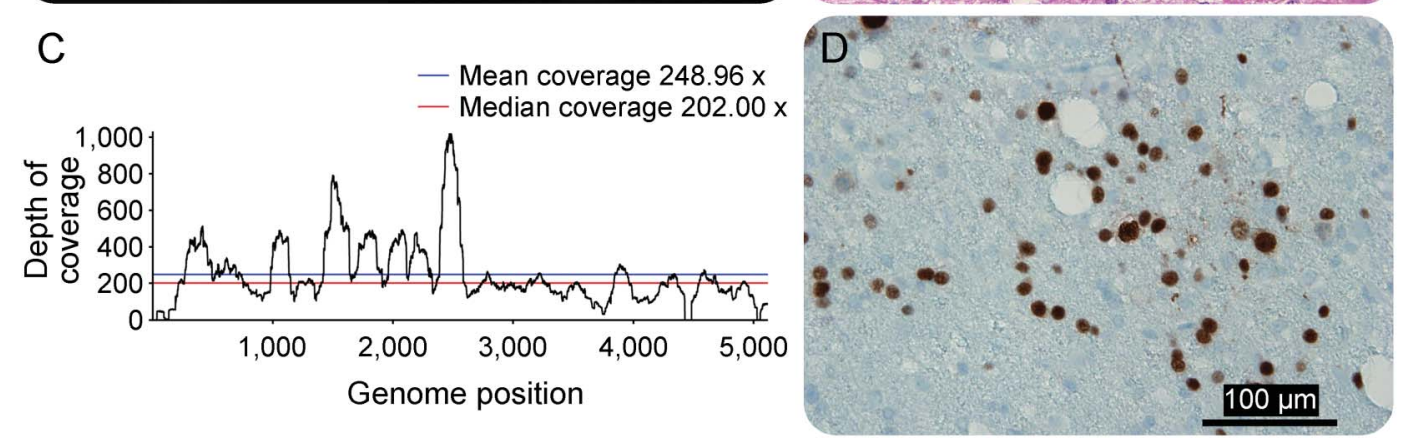

(A) MRI in patient PT-5 shows an extensive area of signal abnormalities in both T2-weighted and fluid-attenuated inversion recovery (FLAIR) MRI sequences that involved the white matter of the left posterior region of the frontal-parietal lobes. (B) Histologic changes observed in hematoxylin \& eosin stain demonstrated extensive gliosis, presence of gemystocytic astrocytes, and mild inflammatory reaction. (C) Next-generation sequencing (NGS) analysis reveals 8,944 JC polyomavirus reads out of 8,954 reads from all viruses. The whole genome of $\mathrm{JC}$ polyomavirus was covered by the reads, at an average depth over 200. The NGS findings are consistent with a diagnosis of progressive multifocal leukoencephalopathy (PML). (D) Immunostaining with SV-40 antibodies, a surrogate for JC virus identification, shows several immunopositive nuclei as confirmation of JC virus infection. These findings confirm the diagnosis of PML.

Corynebacterium kroppenstedtii reads were seen, higher (45\% of nonhuman and noncontaminant reads) than in any other patient (appendix e-2). Although Delftia might have been a contaminant, a combination of antibiotic treatments produced clinical improvement and decreased dural enhancement in brain MRI (figure 5B). It was concluded that the pachymeningeal reaction was possibly associated with a chronic evolving infection possibly triggered by cataract surgery and intraocular lens implantation although no confirmatory studies were obtained to link the potential role of Delftia or other bacteria in the pathogenesis of pachymeningitis.

Patient PT-7 (Fanconi anemia, neurodevelopmental disorder, and cerebritis). A 19-year-old man developed new-onset right-sided weakness and seizures. A brain MRI demonstrated a left-sided brain mass (figure e-1) and a brain biopsy suggested the diagnosis of lymphoma. He was treated with IV and intrathecal dexamethasone via an Ommaya reservoir. The patient deteriorated clinically and was transferred to our institution. A second biopsy of the brain lesion showed chronic inflammation (figure e-1) and coagulative necrosis. Microbiological studies were negative. NGS analysis of the biopsy did not reveal a clear candidate, but did show an unusually high presence of Lactococcus lactis, a common additive in dairy products that rarely causes human infections. A total of 244 reads mapped to the genus Lactococcus, 201 of which were specific to $L$ lactis cremoris.

However, 1 year later (March 2016), re-analysis of this patient's data revealed 429 reads from Elizabethkingia, a newly emerging pathogen that caused significant morbidity in a cluster of cases in Wisconsin. ${ }^{20}$ No more than 2 reads from this species were detected in any other sample. The genomes for this species were unavailable at the time of the original analysis, but 3 genomes are now available: E sp BM10, $E$ anophelis NUHP1, and E meningoseptica FMS-007. The greatest number of matches in this sample was to Esp BM10.

Cases with nonspecific or negative findings that were clinically useful. Sequencing yielded no specific findings to support a diagnosis of infection in 5 cases (table 1). In case PT-3, a 23-year-old woman with status epilepticus following a febrile illness was diagnosed with febrile infection-related epilepsy syndrome (FIRES), ${ }^{21}$ after extensive microbiologic and NGS studies were negative for pathogens. In 

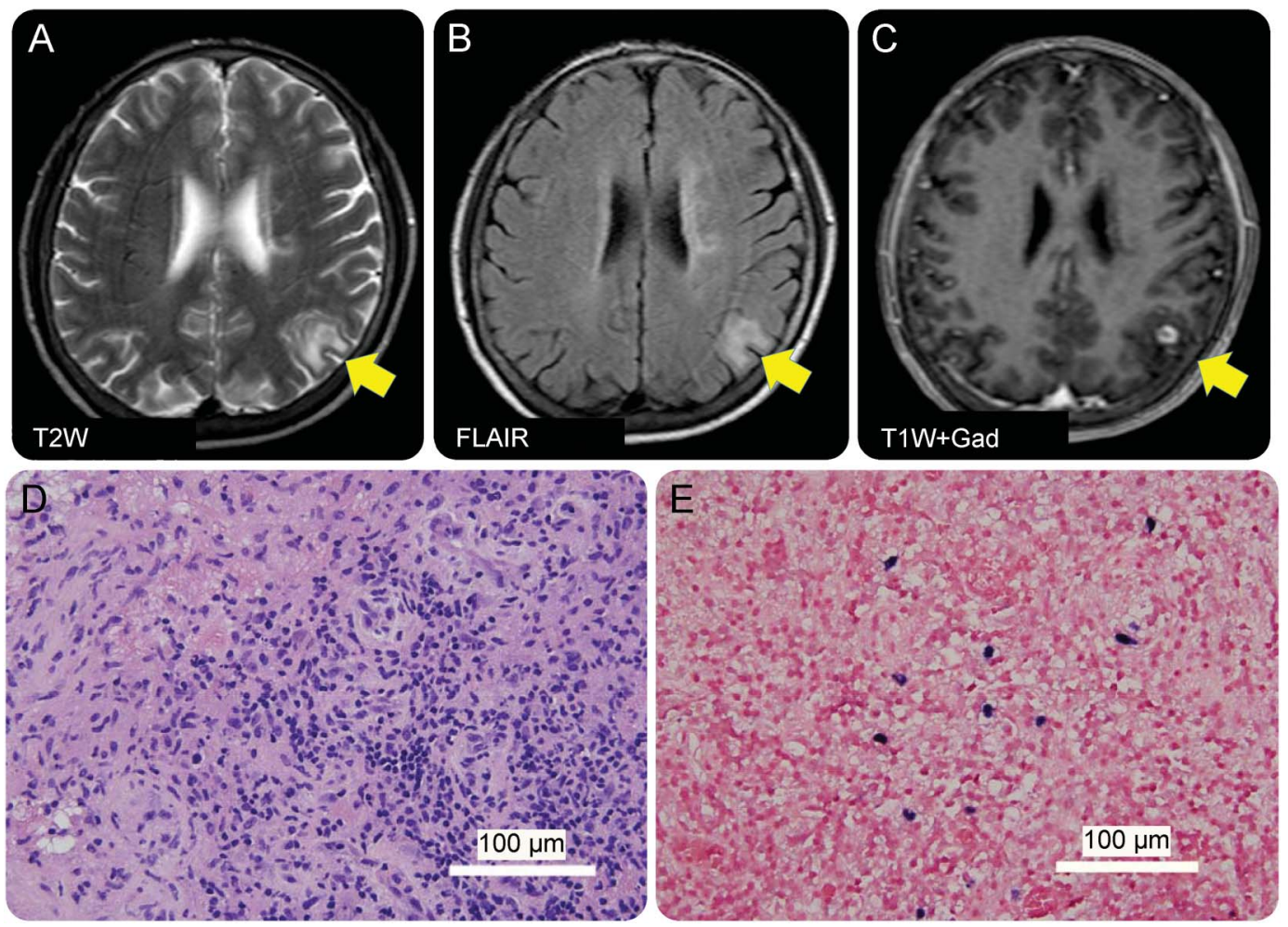

(A-C) Brain MRI shows a focal area of cortical and subcortical signal intensity abnormality. T1-weighted sequences enhanced with gadolinium show a ring-enhanced lesion with perilesional edema, which was the target for the brain biopsy. (D) Histologic demonstration of a granulomatous and lymphohistocytic inflammation with foci of necrosis (hematoxylin \& eosin stain) in patient PT-10. (E) In situ hybridization for EBV-encoded RNA in the brain biopsy tissues, which validates the presence of EBV as it had been established by next-generation sequencing and confirms the diagnosis of EBV encephalitis. FLAIR = fluid-attenuated inversion recovery.

cases PT-4 and PT-9, suspected to have granulomatous inflammation or sarcoidosis based on brain MRI and biopsy findings, NGS showed no evidence of specific infection. Subsequent steroid treatment was associated with improvement in both cases. In 2 cases, neuropathologic studies demonstrated that the disease process was associated with glial tumors despite initial concerns about infections. In 1 patient, PT-6, DNA sequencing identified 15 viral reads mapped to JCV, a finding considered to be incidental, although JCV has been implicated in the pathogenesis of astrocytomas in nonhuman primates. ${ }^{22}$

DISCUSSION We describe the power of large-scale unbiased sequencing along with computational analysis as a diagnostic tool to establish the presence or absence of active infection in brain biopsies from patients with neuroinflammatory disorders suspected to be associated with pathogenic microorganisms. With current technology, the minimum time required for sequencing is approximately 1 day, with initial analysis requiring less than an hour after the sequencing is complete. The current clinical standards for neuropathologic diagnosis of infections, which include histologic, immunologic, and selected molecular techniques (e.g., PCR, 16S rRNA, in situ hybridization), have limited power even when specific neurologic infectious disorders are suspected.

In our study, NGS facilitated the identification of a pathogenic agent in 3 patients for whom the presence of such agent was further validated by immunohistologic or molecular techniques. In these 5 patients, standard diagnostic studies had failed to define etiologic agents. In case PT-8, in which $M$ tuberculosis was identified by NGS, the findings of sequencing studies along with brain pathology features of necrotizing granuloma supported treatment that led to clinical improvement. Similarly in cases PT-5 and PT-10, the confidence in the findings of JCV and EBV was strengthened by immunocytochemical and in situ hybridization techniques, which further supported the finding of a pathogenic role of these viruses. Although PCR studies for EBV and JCV in CSF are well-established in clinical practice, 


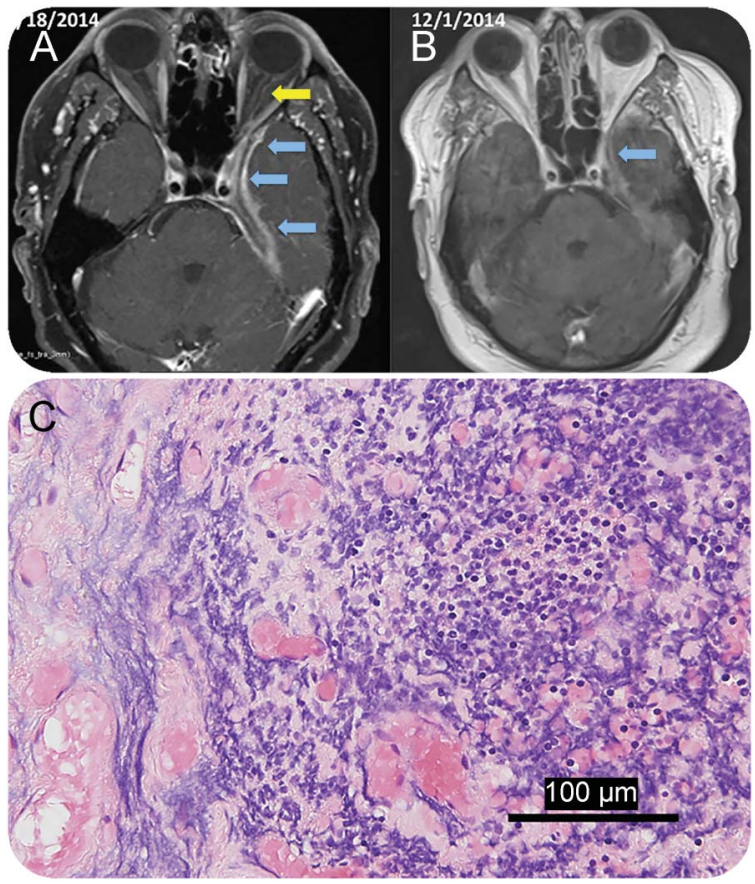

(A) Neuroimaging studies in patient PT-2 demonstrate the presence of pachymeningeal and leptomeningeal enhancement (blue arrows) localized in the medial aspect of the left middle cranial fossae extending to the orbital apex with involvement of the dural margin of the left cavernous sinus, Meckel cave, and foramen ovale. Enhancement of the left optic nerve dura (yellow arrow) is also noted in the March 18, 2014, MRI. (B) Neuroimaging studies following 5 weeks of antibiotic treatment (December 1, 2014) show decreased meningeal and dural enhancement along the anteromedial left temporal lobe margin and tentorial leaflet as well as optic nerve dural sheath. (C) Histopathologic studies of the dura and adjacent bone show chronic inflammation comprising lymphocytes, macrophages, and a few plasma cells (hematoxylin \& eosin stain).

there are specific clinical situations in which brain biopsies are required. For example, the presence of EBV DNA in CSF of immunocompromised patients should be interpreted with caution as EBV may coexist with other opportunistic infections. ${ }^{23}$ In case PT10, for which CSF PCR studies were negative, the finding of EBV by NGS validated by EBV-encoded RNA in situ hybridization helped to establish a diagnosis of EBV-associated encephalitis and also counteracted the brain MRI evidence, which was suggestive of toxoplasmosis. This case shows many similarities to a previous report ${ }^{24}$ of $\mathrm{EBV}$-induced brain lesions in a patient following organ transplantation. Similarly, the value of metagenomic sequencing in the diagnosis of PT-5 was demonstrated by the inconclusiveness of 2 prebiopsy qualitative JCV-PCR studies in CSF. Although pathology and PCR techniques might have helped to establish the diagnosis of PML, case PT-5 represents a good proof-of-concept that NGS studies are useful and may provide better characterization of the JCV genome for prognosis purposes. ${ }^{25,26}$

Although a high degree of certainty could not be achieved in some cases, NGS provided information that facilitated a better understanding of the high or

low likelihood of CNS infection in the other 5 patients. In 2 cases, NGS findings were considered indeterminate as suspected microorganisms frequently seen as contaminants were identified although their pathogenic role could not be confirmed by histology, cultures, or direct pathogen visualization. In these 2 patients, the metagenomic analysis yielded a high number of reads of bacteria frequently recognized as environmental contaminants such as $D$ acidovorans and $C$ kroppenstedtii (PT-2), normal commensals of skin, and L lactis cremoris and Elizabethkingia (PT-7). Although these bacteria might had entered the CNS by surgical procedures such as ophthalmologic surgery (PT-2) and Ommaya reservoir (PT-7), the validation that these assumed contaminants were pathogenic organisms by other microbiologic or morphologic methods was difficult to obtain, for which the NGS findings were considered indeterminate. Although these environmental contaminants have been associated with neuropathologic conditions ${ }^{27-29}$ and outbreak of illness, ${ }^{20}$ our findings expose one of the limitations in the interpretation of NGS, in that the demonstration that these presumed contaminants were pathogenic organisms would require a detailed validation approach to prove pathogenicity.

Whereas microbiome analysis of brain biopsies was able to identify definite or possible pathogenic microorganisms in 5 of 10 cases, no pathogenic species were identified in the other 5 cases. Negative NGS studies may help support a finding that a brain infection is not present, although the negative predictive value of sequencing assays is not yet known. In case PT-3, the failure to demonstrate a pathogen helped to determine her neurologic disorder was most likely associated with FIRES, a rare condition of uncertain etiology in which infection is suspected. ${ }^{21}$ In another 2 cases, PT- 4 and PT-9, NGS showed no clear evidence of pathogenic microorganisms associated with the granulomatous inflammation noted on brain biopsies. We therefore determined there was no active infection, a finding that was further supported by the beneficial effect of steroid treatment. These 2 cases demonstrate the way in which negative NGS findings may help clinicians to decrease concerns about an active infection, as the presence of granulomatous inflammation always raises concerns about the presence of atypical bacteria, mycobacteria, or fungal infections in which the use of steroids would be deleterious. Clearly, microbiome analysis of brain tissues has limitations, and the absence of pathogenic microorganisms should be interpreted with caution. It is possible that the timing or site of the brain biopsy may not coincide with the period of active infection, and thus 
inflammatory changes could represent reactive immune responses to an earlier or nearby infection. Negative NGS studies in patients with neuroinflammatory diseases may also suggest the cause of such disorders is due to postinfectious or autoimmune inflammatory responses rather than an active infection.

Sequence-based results for patients in whom NGS studies were negative or inconclusive, as well as in cases supporting specific diagnoses, contained common skin bacteria and other contaminants that were not considered clinically relevant (appendix e-1). While we cannot rule out these species as pathogens, their presence in multiple samples collected from different patients widely spaced apart in time indicates that they are not likely to be causally related to the patients' symptoms. For example, we observed the skin bacterium Propionibacterium acnes in almost all samples, illustrating the difficulty of capturing a pure sample without any microbial spillover from the intervening tissues. These findings illustrate the difficulty and complexity of identifying pathogens in human samples, which unavoidably contain some bacterial species because the human body is not a sterile environment and brain biopsies are obtained via skin incision, which is colonized with multiple bacteria. ${ }^{30}$ In addition, surgical procedures that include the use of irrigation solutions, biopsy handling, as well as processing methods (e.g., DNA extraction kits and other sequencing reagents) often contain bacterial DNA that may be captured by NGS methods especially when the concentration of microbial DNA is low. ${ }^{31}$

An important limitation of computational analysis in microbiome studies is the size and completeness of genome sequence databases. Although thousands of bacterial and viral species have been sequenced, many others including human pathogens may yet be discovered, and unknown pathogens cannot be detected until their genomes are sequenced. Eukaryotic pathogens represent a larger challenge: many of these remain to be sequenced, and even those that have been sequenced are available only in draft form, with many gaps and errors in the publicly available genomes.

We described here the power of large-scale unbiased sequencing along with computational-based metagenomic analysis in the study of brain biopsies. Our study opens the possibility for more extensive use of NGS techniques in the identification of pathogens in patients with suspected CNS infections or neuroinflammatory disorders.

\section{AUTHOR CONTRIBUTIONS}

Steven L. Salzberg: study concept or design, computational biology data analysis, study supervision, drafting/revising the manuscript, obtaining funding. Florian P. Breitwieser: computational biology data analysis, revising manuscript. Anupama Kumar: tissue processing, clinical data analysis, revising manuscript. Haiping Hao: tissue processing, DNA/RNA sequencing. Peter Burger: neuropathology analysis, revising manuscript. Fausto J. Rodriguez: neuropathology analysis, revising manuscript. Michael Lim: neurosurgical procedure, revising manuscript. Alfredo Quiñones-Hinojosa: neurosurgical procedure, revising manuscript. Gary L. Gallia: neurosurgical procedure, revising manuscript. Jeffrey A. Tornheim: clinical data analysis, revising manuscript. Michael T. Melia: clinical data analysis, revising manuscript. Cynthia L. Sears: clinical data analysis, revising manuscript. Carlos A. Pardo: study concept or design, neuropathology analysis, clinical data analysis, study supervision, drafting/revising the manuscript, obtaining funding.

\section{ACKNOWLEDGMENT}

The authors thank Drs. Arun Venkatasen, Matthew Ippolito, and Robert McMillan for contributing ideas and patient care.

\section{STUDY FUNDING}

Supported in part by the NIH under grant R01 HG006677 (S.L.S.) by the US Army Research Office under grant number W911NF-14-1-0490 (S.L.S.) and The Bart McLean Fund for Neuroimmunology ResearchJohns Hopkins Project Restore (C.A.P.).

\section{DISCLOSURE}

S.L. Salzberg is an Associate Editor, Editorial Board member, or Editorial Advisory Board member for the following journals: Genome Biology, PeerJ Computer Science, BMC Biology, Biology Direct, Journal of Computational Biology, BMC Genomics, BMC Bioinformatics, and Evolutionary Bioinformatics; and received research support from NIH. F.P. Breitwieser, A. Kumar, H. Hao, and P. Burger report no disclosures. F.J. Rodriguez receives publishing royalties from Elsevier. M. Lim served on the scientific advisory boards for Novartis-DSMB, BMS, and Stryker; received travel funding and speaker honoraria from Stryker and BMS; holds a patent for the combination of focused radiation and immunotherapy; receives publishing royalties for Handbook of Radiosurgery; performs neurosurgery; and received research support from Stryker, BMS, Aegenus, CellDex, and Arbor. A. Quinones-Hinojosa received travel funding and speaker honoraria from Speak Inc. and American Program Bureau, Inc.; is on the editorial board for Cancer Today; holds patents for Microfluid assay for the determination of tumor cell metastatic propensity, A novel, high-throughput, nanotopographic platform for screening cell migratory behavior, Quantitative tissue property mapping for real time tumor detection and interventional guidance, Nanoparticle modification of human adipose-derived mesenchymal stem cells for brain cancer and other neurological diseases, Hydrogels for local treatment of brain-related diseases, Methods for phenotypic classification of cells based on migratory behavior, Use of AGX/UAP inhibitors to inhibit flux through the hexosamine biosynthetic pathway, Self-assembling verteporfin amphiphiles (SAVA) for local brain cancer therapy, Predicting brain tumor patient prognosis, and NAAG as an indicator of clinical prognosis for glioblastoma; receives publishing royalties from Elsevier, UC Press, LID Espanol, Thieme, Stem Cells International, and Jaypee Brothers Medical Publishers; has consulted for Stryker and Integra; is on the speaker bureau for Speak, Inc., and American Program Bureau, Inc.; and received research support from Stryker, Integra, NIH, Johns Hopkins University, JHU Coulter Foundation, Maryland Stem Cell Research Fund (TEDCO), Maryland Biotechnology Development Award, and Allegheny Health Network-Johns Hopkins Cancer Research Fund. G.L. Gallia received research support from Chordoma. J.A. Tornheim reports no disclosures. M.T. Melia's institution received research support from Merck, Sharpe \& Dohme, Janssen, Gilead, Bristol-Myers Squibb, and AbbVie; and he received research support from NIH, CDC, and Johns Hopkins School of Medicine. C.L. Sears is an associate editor for Clinical Infectious Diseases; holds a patent for biofilms in colon cancer as a diagnostic approach to identify individuals at risk for colon cancer; receives royalties from Up-to-Date; and received research support from NIH, Johns Hopkins, and Institut Merieux. C.A. Pardo received research support from Accorda Pharmaceuticals, NIH/National 
Institute of Neurological Disorders and Stroke, and Bart McLean Fund for Neuroimmunology Research. Go to Neurology.org/nn for full disclosure forms.

Received February 4, 2016. Accepted in final form May 9, 2016.

\section{REFERENCES}

1. Glaser CA, Honarmand S, Anderson LJ, et al. Beyond viruses: clinical profiles and etiologies associated with encephalitis. Clin Infect Dis 2006;43:1565-1577.

2. Barzon L, Lavezzo E, Militello V, Toppo S, Palu G. Applications of next-generation sequencing technologies to diagnostic virology. Int J Mol Sci 2011;12: 7861-7884.

3. Yin L, Liu L, Sun Y, et al. High-resolution deep sequencing reveals biodiversity, population structure, and persistence of HIV-1 quasispecies within host ecosystems. Retrovirology 2012;9:108.

4. Lecuit M, Eloit M. The human virome: new tools and concepts. Trends Microbiol 2013;21:510-515.

5. Wylie KM, Weinstock GM, Storch GA. Virome genomics: a tool for defining the human virome. Curr Opinion Microbiol 2013;16:479-484.

6. Chan BK, Wilson T, Fischer KF, Kriesel JD. Deep sequencing to identify the causes of viral encephalitis. PLoS One 2014;9:e93993.

7. Wilson MR, Shanbhag NM, Reid MJ, et al. Diagnosing balamuthia mandrillaris encephalitis with metagenomic deep sequencing. Ann Neurol 2015;78:722-730.

8. Naccache SN, Peggs KS, Mattes FM, et al. Diagnosis of neuroinvasive astrovirus infection in an immunocompromised adult with encephalitis by unbiased next-generation sequencing. Clin Infect Dis 2015;60:919-923.

9. Brown JR, Morfopoulou S, Hubb J, et al. Astrovirus VA1/HMO-C: an increasingly recognized neurotropic pathogen in immunocompromised patients. Clin Infect Dis 2015;60:881-888.

10. Fremond ML, Perot P, Muth E, et al. Next-generation sequencing for diagnosis and tailored therapy: a case report of astrovirus-associated progressive encephalitis. J Pediatr Infect Dis Soc 2015;4:e53-e57.

11. Greninger AL, Messacar K, Dunnebacke T, et al. Clinical metagenomic identification of Balamuthia mandrillaris encephalitis and assembly of the draft genome: the continuing case for reference genome sequencing. Genome Med 2015;7:113.

12. Tan le V, van Doorn HR, Nghia HD, et al. Identification of a new cyclovirus in cerebrospinal fluid of patients with acute central nervous system infections. MBio 2013;4: e00231-e00313.

13. Wylie KM, Blanco-Guzman M, Wylie TN, et al. Highthroughput sequencing of cerebrospinal fluid for diagnosis of chronic Propionibacterium acnes meningitis in an allogeneic stem cell transplant recipient. Transpl Infect Dis 2016;18:227-233.

14. Hoffmann B, Tappe D, Hoper D, et al. A variegated squirrel bornavirus associated with fatal human encephalitis. N Engl J Med 2015;373:154-162.
15. Wilson MR, Naccache SN, Samayoa E, et al. Actionable diagnosis of neuroleptospirosis by next-generation sequencing. N Engl J Med 2014;370:2408-2417.

16. Wood DE, Salzberg SL. Kraken: ultrafast metagenomic sequence classification using exact alignments. Genome Biol 2014;15:R46.

17. Altschul SF, Madden TL, Schaffer AA, et al. Gapped BLAST and PSI-BLAST: a new generation of protein database search programs. Nucleic Acids Res 1997;25:3389-3402.

18. Langmead B, Salzberg SL. Fast gapped-read alignment with Bowtie 2. Nat Methods 2012;9:357-359.

19. Ferenczy MW, Marshall LJ, Nelson CD, et al. Molecular biology, epidemiology, and pathogenesis of progressive multifocal leukoencephalopathy, the JC virus-induced demyelinating disease of the human brain. Clin Microbiol Rev 2012;25:471-506.

20. CDC. Outbreaks: multistate outbreak of infections caused by Elizabethkingia anophelis [online]. Available at: http:// www.cdc.gov/elizabethkingia/outbreaks/index.html. Accessed May 2, 2016.

21. Pardo CA, Nabbout R, Galanopoulou AS. Mechanisms of epileptogenesis in pediatric epileptic syndromes: Rasmussen encephalitis, infantile spasms, and febrile infectionrelated epilepsy syndrome (FIRES). Neurotherapeutics 2014;11:297-310.

22. Maginnis MS, Atwood WJ. JC virus: an oncogenic virus in animals and humans? Semin Cancer Biol 2009;19:261-269.

23. Martelius T, Lappalainen M, Palomaki M, Anttila VJ. Clinical characteristics of patients with Epstein Barr virus in cerebrospinal fluid. BMC Infect Dis 2011;11:281.

24. Khalil M, Enzinger C, Wallner-Blazek M, et al. EpsteinBarr virus encephalitis presenting with a tumor-like lesion in an immunosuppressed transplant recipient. J Neurovirol 2008; 14:574-578.

25. Ryschkewitsch CF, Jensen PN, Major EO. Multiplex qPCR assay for ultra sensitive detection of JCV DNA with simultaneous identification of genotypes that discriminates non-virulent from virulent variants. J Clin Virol 2013;57: 243-248.

26. Marshall LJ, Ferenczy MW, Daley EL, Jensen PN, Ryschkewitsch CF, Major EO. Lymphocyte gene expression and JC virus noncoding control region sequences are linked with the risk of progressive multifocal leukoencephalopathy. J Virol 2014;88:5177-5183.

27. Feierabend D, Reichart R, Romeike B, Kalff R, Walter J. Cerebral abscess due to Lactococcus lactis cremoris in a child after sinusitis. Clin Neurol Neurosurg 2013;115:614-616.

28. Inoue $\mathrm{M}$, Saito $\mathrm{A}$, Kon $\mathrm{H}$, et al. Subdural empyema due to Lactococcus lactis cremoris: case report. Neurol Med Chir 2014;54:341-347.

29. Topcu Y, Akinci G, Bayram E, Hiz S, Turkmen M. Brain abscess caused by Lactococcus lactis cremoris in a child. Eur J Pediatr 2011;170:1603-1605.

30. Grice EA, Segre JA. The skin microbiome. Nat Rev Microbiol 2011;9:244-253.

31. Salter SJ, Cox MJ, Turek EM, et al. Reagent and laboratory contamination can critically impact sequence-based microbiome analyses. BMC Biol 2014;12:87. 


\title{
Neurology \\ Neuroimmunology \& Neuroinflammation
}

\author{
Next-generation sequencing in neuropathologic diagnosis of infections of the nervous \\ system \\ Steven L. Salzberg, Florian P. Breitwieser, Anupama Kumar, et al. \\ Neurol Neuroimmunol Neuroinflamm 2016;3; \\ DOI 10.1212/NXI.0000000000000251
}

This information is current as of June 13, 2016

\section{Updated Information \& \\ Services}

Supplementary Material

References

Citations

Subspecialty Collections

Permissions \& Licensing

Reprints including high resolution figures, can be found at:

http://nn.neurology.org/content/3/4/e251.full.html

Supplementary material can be found at:

http://nn.neurology.org/content/suppl/2016/06/13/3.4.e251.DC1

http://nn.neurology.org/content/suppl/2016/08/15/3.4.e251.DC2

This article cites 30 articles, 2 of which you can access for free at: http://nn.neurology.org/content/3/4/e251.full.html\#\#ref-list-1

This article has been cited by 9 HighWire-hosted articles:

http://nn.neurology.org/content/3/4/e251.full.html\#\#otherarticles

This article, along with others on similar topics, appears in the following collection(s):

All Infections

http://nn.neurology.org//cgi/collection/all_infections

Information about reproducing this article in parts (figures,tables) or in its entirety can be found online at:

http://nn.neurology.org/misc/about.xhtml\#permissions

Information about ordering reprints can be found online:

http://nn.neurology.org/misc/addir.xhtml\#reprintsus

Neurol Neuroimmunol Neuroinflamm is an official journal of the American Academy of Neurology.

Published since April 2014, it is an open-access, online-only, continuous publication journal. Copyright $\odot$ 2016 American Academy of Neurology. All rights reserved. Online ISSN: 2332-7812.

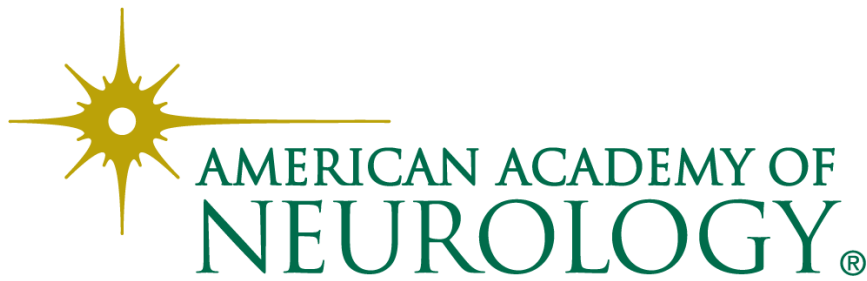

